\title{
Asymptotic nonlocality
}

\author{
Jens Boos ${ }^{*}$ and Christopher D. Carone ${ }^{\dagger}$ \\ High Energy Theory Group, Department of Physics, William \& Mary, \\ Williamsburg, Virgina 23187-8795, USA
}

(Received 26 April 2021; accepted 5 July 2021; published 22 July 2021)

\begin{abstract}
We construct a theory of real scalar fields that interpolates between two different theories: a Lee-Wick theory with $N$ propagator poles, including $N-1$ Lee-Wick partners, and a nonlocal infinite-derivative theory with kinetic terms modified by an entire function of derivatives with only one propagator pole. Since the latter description arises when taking the $N \rightarrow \infty$ limit, we refer to the theory as "asymptotically nonlocal." Introducing an auxiliary-field formulation of the theory allows one to recover either the higherderivative form (for any $N$ ) or the Lee-Wick form of the Lagrangian, depending on which auxiliary fields are integrated out. The effective scale that regulates quadratic divergences in the large- $N$ theory is the would-be nonlocal scale, which can be hierarchically lower than the mass of the lightest Lee-Wick resonance. We comment on the possible utility of this construction in addressing the hierarchy problem.
\end{abstract}

DOI: 10.1103/PhysRevD.104.015028

\section{INTRODUCTION}

When the Higgs boson was discovered at the Large Hadron Collider (LHC) in 2012 at a mass of $125 \mathrm{GeV}$, an immediate question was this: why is it so light compared to the Planck scale at $10^{19} \mathrm{GeV}$ ? The standard model, in its present form, does not provide a mechanism that protects the Higgs mass from picking up large radiative corrections from Planck-scale physics. And if there was no such mechanism, then the observed Higgs mass had to be the result of an incredibly fine-tuned almost cancellation between the bare mass and its corrections.

This has been dubbed the "hierarchy problem," and while there have been many proposed solutions, in this paper we would like to focus on the Lee-Wick approach [1]. The Lee-Wick standard model [2] provides an inherent mechanism to address the hierarchy problem: supplementing the spectrum of the standard model by $\mathrm{TeV}$-scale LeeWick partner particles with wrong-sign kinetic and mass terms leads to a cancellation of quadratic divergences in scalar self-energies, with the precise spectrum of Lee-Wick resonances left for experiments to determine. These models have been shown to be unitary, provided certain prescriptions are adopted in momentum space to deal with the different pole structure [3] (see also [4]), are causal at the

\footnotetext{
jboos@wm.edu

cdcaro@wm.edu
}

Published by the American Physical Society under the terms of the Creative Commons Attribution 4.0 International license. Further distribution of this work must maintain attribution to the author(s) and the published article's title, journal citation, and DOI. Funded by SCOAP. macroscopic level [5], have subsequently been generalized to include additional partner particles [6,7], and have garnered much attention in the phenomenological literature as well [8]. However, to date, no such Lee-Wick partner particles have been found up to $\sqrt{s} \sim \mathcal{O}(10) \mathrm{TeV}$ [9], and while shifting these masses to higher, unprobed energies would in principle be permissible, it would no longer provide a convincing solution of the hierarchy problem.

The last decades have also seen the development of nonlocal field theories [10-15], in particular those without additional degrees of freedom, sometimes referred to as "ghost-free" theories. While these Lorentz-invariant nonlocal theories were originally intended as simple generalizations of Pauli-Villars regularization schemes [16-18] they have since been rediscovered in $p$-adic string theory and noncommutative geometry [19-22]. A large body of research has been devoted to a better understanding of $a b$ initio nonlocal theories, and recent work and applications include regular bouncing cosmologies [23], nonlocality in string theory [24,25], regularization of the gravitational field via nonlocality [26-28], as well as the role of the Wick rotation vis-à-vis unitarity and causality [29-34]. For a review of infinite-derivative quantum field theory see Ref. [35], and for additional references we refer the reader to Ref. [36].

In nonlocal theories, the scale of nonlocality is set by a free parameter that we will call $M_{\mathrm{nl}}$; in ghost-free models, this scale does not correspond to the mass of a resonance as there are no corresponding poles in the propagator. Given the current status of LHC searches for new particles, this is potentially a desirable property for a realistic nonlocal extension of the standard model. One might expect that nonresonant effects on scattering amplitudes would allow 
$M_{\mathrm{nl}}$ to be as low as the typical, $\mathrm{TeV}$-scale collider bounds on new physics that does not violate flavor, $C P$ or Lorentz invariance. In the present work, we develop a simple scalar toy model that interpolates between a Lee-Wick and a nonlocal theory, where the latter is obtained as the number of Lee-Wick resonances tends to infinity and the spectrum of heavy particles decouples. What is interesting about this "asymptotically nonlocal" theory is that the would-be nonlocal scale, $M_{\mathrm{nl}}$, becomes the effective regulator of scalar loop diagrams, while remaining hierarchically lower than the mass of the lightest Lee-Wick resonance. (For another proposal in which the effective scale of nonlocality is expected to be much lower than a cutoff scale see [37].) In the case where the number of Lee-Wick resonances is finite but large, the hierarchy problem in this particular model is resolved even as the Lee-Wick resonances are decoupled from the low-energy effective theory.

It should be stressed that our goal is to construct specific Lee-Wick theories with $N$ propagator poles that have the decoupling and divergence properties described above. These higher-derivative theories are strictly local and avoid a pathology of the nonlocal theory that they approach, namely, the inability to Wick rotate in Minkowski space leading to a loss of unitarity [29-34]. Our finite- $N$ LeeWick theories are treated as fundamental and are of interest in their own right; the significance of the $N \rightarrow \infty$ limiting form is only in that it suggests an organizing principle that assures the emergence of a derived mass scale that regulates loop integrals, one that is hierarchically smaller than the lightest Lee-Wick partner mass. Our approach is in some sense analogous to dimensional deconstruction [38,39], where interesting four-dimensional theories can be defined whose properties may be understood in terms of a fivedimensional limiting theory, even though one is never actually interested in taking or exactly reproducing the limit. Just as a deconstructed model should not be thought of as a truncation of a five-dimensional theory for the purposes of approximation, the same perspective applies to the finite $N$ theories that we are about to present.

This paper is organized as follows: in Sec. II, we develop our basic framework of an auxiliary field theory, and prove that integrating out the auxiliary fields results in an effectively higher-derivative theory, and-in the limit of infinitely many auxiliary fields - results in a manifestly nonlocal theory; we refer to this as asymptotic nonlocality. In Sec. III, we match this theory to a Lee-Wick theory, and as an application in Sec. IV we compute the scalar selfenergy at finite and infinite $N$, at one loop. We show for this simple model that asymptotic nonlocality can indeed separate the scale of the lightest Lee-Wick resonances from the scale that characterizes the convergence of loop diagrams. We summarize our findings in Sec. V and suggest that no impediments exist to generalizing this framework to Abelian gauge theories, non-Abelian gauge theories, and, in principle, the entire standard model.

\section{FRAMEWORK}

Consider the following (nongeneric) theory of $N$ real scalar fields $\phi_{j}$, and $N-1$ real scalar fields $\chi_{j}$ :

$\mathcal{L}_{N}=-\frac{1}{2} \phi_{1} \square \phi_{N}-V\left(\phi_{1}\right)-\sum_{j=1}^{N-1} \chi_{j}\left[\square \phi_{j}-\left(\phi_{j+1}-\phi_{j}\right) / a_{j}^{2}\right]$.

Here, the $a_{j}$ are $N-1$ constants with dimension of length, and we have set all the coefficients of the terms involving the $\chi_{i}$ to one, for simplicity. The signature of the metric is $(+,-,-,-)$ and $\square \equiv \partial_{\mu} \partial^{\mu}$. Functionally integrating over the $\chi_{i}$ leads to the constraints

$\square \phi_{j}-\left(\phi_{j+1}-\phi_{j}\right) / a_{j}^{2}=0, \quad$ for $j=1 \ldots N-1$.

Equation (2.2) is exact at the quantum level, since functionally integrating over the $\chi_{j}$, which appear linearly in the Lagrangian, yields a functional delta function in the generating functional for the correlation functions of the theory. ${ }^{1}$ This delta function allows one to perform the functional integration over $N-1$ of the $\phi_{j}$ fields, which we will take as those with $j=2 \ldots N$; this yields a theory for $\phi_{1}$. It is instructive first to consider the case where the $a_{j}$ are all equal, i.e., $a_{j} \equiv a$. Then recursive application of Eq. (2.2) implies

$$
\phi_{N} \equiv\left(1+\frac{\ell^{2} \square}{N-1}\right)^{N-1} \phi_{1},
$$

where $\ell^{2} \equiv(N-1) a^{2}$. Letting $N \rightarrow \infty$ while holding $\ell$ fixed one finds

$$
\phi_{\infty}=e^{\ell^{2} \square} \phi_{1},
$$

such that the resulting Lagrangian takes the form

$$
\mathcal{L}_{\infty}=-\frac{1}{2} \phi_{1} \square e^{\ell^{2} \square} \phi_{1}-V\left(\phi_{1}\right) .
$$

This is a familiar form found in the literature on nonlocal quantum field theories, with the scale of nonlocality fixed by the parameter $\ell$. It is important to note that the $\square$ operator appearing in the exponential is not necessarily the best choice for a nonlocal theory defined in Minkowski spacetime due to time-dependent instabilities [43,44]. However, our construction holds if one replaces the $\chi_{j} \square \phi_{j}$ terms in Eq. (2.1) with $\chi_{j} \mathcal{D} \phi_{j}$, where $\mathcal{D}$ can be

\footnotetext{
${ }^{1}$ Note that taking the $a_{j} \rightarrow 0$ limit in Eq. (2.2) transforms the difference $\phi_{j+1}-\phi_{j}$ into a derivative with respect to a new parameter; the approach of exchanging nonlocality for locality in a higher-dimensional space has been explored in Refs. [40,41]; see also [42].
} 
any $\chi_{j}$-independent differential operator that one would like to appear in the exponential. Inter alia, this includes the Lorentz-violating possibility that $\mathcal{D}=\vec{\nabla}^{2}$, discussed in Ref. [45].

The choice of a single $a_{j} \equiv a$ is convenient for the purpose of illustration, but it has the following drawback: at finite $N$, the propagator of the theory,

$$
D_{F}\left(p^{2}\right)=\frac{i}{p^{2}\left(1-a^{2} p^{2}\right)^{N-1}},
$$

cannot be written as a sum over simple poles. [Here we have assumed, for simplicity, that there is no $\phi_{1}$ mass term in the potential $V\left(\phi_{1}\right)$.] Alternatively, Eq. (2.6) can be written as a sum over simple poles, but only in a limit where the wave function renormalization of all but one factor diverges. In what follows, we avoid this problem by assuming that the $a_{j}$ are nondegenerate. The higherderivative form of the theory is then given by

$$
\mathcal{L}_{N}=-\frac{1}{2} \phi_{1} \square\left[\prod_{j=1}^{N-1}\left(1+\frac{\ell_{j}^{2} \square}{N-1}\right)\right] \phi_{1}-V\left(\phi_{1}\right),
$$

where $\ell_{j}^{2} \equiv(N-1) a_{j}^{2}$, and where the propagator now takes the form

$$
D_{F}\left(p^{2}\right)=\frac{i}{p^{2}} \prod_{j=1}^{N-1}\left(1-\frac{\ell_{j}^{2} p^{2}}{N-1}\right)^{-1} .
$$

One would expect that the same limiting form of the Lagrangian, Eq. (2.5), is obtained when $N \rightarrow \infty$ and the $\ell_{j}$ approach a common, nonvanishing value. This finite- $N$ theory provides a spectrum of nondegenerate states that alternate between ordinary particles and ghosts [18], i.e., particles whose propagators have wrong-sign residues. This is what one would expect in Lee-Wick theories that have additional higher-derivative quadratic terms [7] beyond those found in the Lee-Wick standard model [2], see also [11]. The physics of the theory at large but finite $N$ is not strictly equivalent to that of the limit $N \rightarrow \infty$ where additional pathologies arise (see Sec. I). Nevertheless, by working with local theories at finite $N$, we inherit a desirable feature of the limiting theory, namely a derived scale that regulates loop integrals and that can be hierarchically smaller than the mass scale of the lightest Lee-Wick partner, $a_{1}^{-1}$. We show this explicitly later.

\section{LEE-WICK FORM OF THE THEORY}

At finite $N$, the theory described in the previous section predicts $N$ physical poles, as can be seen from Eq. (2.8), which follows immediately from the higher-derivative form of the theory for $\phi_{1}$. We obtained this higher-derivative form by integrating out $N-1$ of the $2 N-1$ scalar fields in
Eq. (2.1). The theory can also be written in a form without higher derivatives, in which there is a separate field corresponding to each of the $N$ poles, with canonical quadratic terms up to overall signs; in the case of the Lee-Wick standard model, this has been called the LeeWick form of the theory [2], a terminology we adopt here. The same form can be achieved in the present model by integrating out a different choice of $N-1$ fields that appear linearly in Eq. (2.1). Let us illustrate this in the present section, in the cases where $N=2$ and $N=3$, and comment on generalizations that are required for arbitrary $N$ at the end.

\section{A. Case $N=2$}

For the case of $N=2$, let us represent the field basis by $\Psi \equiv\left(\phi_{1}, \chi_{1}, \phi_{2}\right)$. The quadratic terms in Eq. (2.1) may then be written as

$$
\mathcal{L}_{\text {quad }}=-\frac{1}{2} \Psi^{T}(K \square+M) \Psi,
$$

where the matrices $K$ and $M$ are given by

$$
K=\left(\begin{array}{ccc}
0 & 1 & 1 / 2 \\
1 & 0 & 0 \\
1 / 2 & 0 & 0
\end{array}\right) \text { and } M=\left(\begin{array}{ccc}
0 & 1 / a_{1}^{2} & 0 \\
1 / a_{1}^{2} & 0 & -1 / a_{1}^{2} \\
0 & -1 / a_{1}^{2} & 0
\end{array}\right) \text {. }
$$

We seek an invertible matrix $S_{2}$ such that $K_{0}=S_{2}^{T} K S_{2}$ and $M_{0}=S_{2}^{T} M S_{2}$ are simultaneously diagonal (or as close to it as possible). With the choice

$$
S_{2}=\left(\begin{array}{ccc}
1 & -1 & 0 \\
0 & 1 / 2 & \xi \\
1 & 0 & -2 \xi
\end{array}\right)
$$

where $\xi$ is an arbitrary nonvanishing real parameter, one finds

$$
K_{0}=\left(\begin{array}{ccc}
1 & 0 & 0 \\
0 & -1 & 0 \\
0 & 0 & 0
\end{array}\right) \text { and } M_{0}=\left(\begin{array}{ccc}
0 & 0 & 0 \\
0 & -1 / a_{1}^{2} & 0 \\
0 & 0 & 4 \xi^{2} / a_{1}^{2}
\end{array}\right)
$$

A number of comments are in order. A combination of unitary rotations and rescalings are sufficient to put the kinetic matrix in the form shown in Eq. (3.4). The remaining transformations needed to diagonalize $M$ must preserve the form of $K_{0}$ [for example, an $\mathrm{SO}(1,1)$ rotation in the upper-left two-by-two block]; the matrix $S_{2}$ encodes all of these transformations. The mass of the $\Psi_{3}$ field appears to be dependent on an arbitrary parameter $\xi$, but $K_{0}$ 
reveals that this linear combination of the original fields has no kinetic term and hence does not correspond to a physical degree of freedom. As before, integrating out $N-1$ fields (in this case, the one field $\Psi_{3}$, whose functional integral can be performed exactly) leaves one with a theory with two physical degrees of freedom, an ordinary particle (with vanishing mass by our initial choice), and a Lee-Wick partner with mass $1 / a_{1}^{2}$. This matches our expectation from the higher-derivative form of the theory, including the correct locations of the poles. Note that the form of $S_{2}$ ensures that the potential $V\left(\phi_{1}\right)$ has no dependence on the auxiliary field $\Psi_{3}$.

\section{B. Case $N=3$}

Let us further strengthen our intuition for extracting the Lee-Wick form of the theory by considering the theory with $N=3$. In this case, the kinetic and mass matrices are

$$
\begin{aligned}
K & =\left(\begin{array}{ccccc}
0 & 1 & 0 & 0 & 1 / 2 \\
1 & 0 & 0 & 0 & 0 \\
0 & 0 & 0 & 1 & 0 \\
0 & 0 & 1 & 0 & 0 \\
1 / 2 & 0 & 0 & 0 & 0
\end{array}\right) \text { and } \\
M & =\left(\begin{array}{ccccc}
0 & 1 / a_{1}^{2} & 0 & 0 & 0 \\
1 / a_{1}^{2} & 0 & -1 / a_{1}^{2} & 0 & 0 \\
0 & -1 / a_{1}^{2} & 0 & 1 / a_{2}^{2} & 0 \\
0 & 0 & 1 / a_{2}^{2} & 0 & -1 / a_{2}^{2} \\
0 & 0 & 0 & -1 / a_{2}^{2} & 0
\end{array}\right),
\end{aligned}
$$

in the field basis $\Psi \equiv\left(\phi_{1}, \chi_{1}, \phi_{2}, \chi_{2}, \phi_{3}\right)$. Again, we seek an invertible matrix (called $S_{3}$ ) that performs a simultaneous diagonalization. In this case, only a block diagonalization is possible. With the choice

$$
S_{3}=\left(\begin{array}{ccccc}
1 & -\sqrt{\frac{y}{y-1}} & -\frac{1}{\sqrt{y-1}} & 0 & 0 \\
0 & \frac{1}{2} \sqrt{\frac{y-1}{y}} & 0 & \frac{y-1}{4 \sqrt{y}} & -\xi / 2 \\
1 & 0 & \sqrt{y-1} & -\sqrt{y} & 0 \\
0 & \frac{1}{2 \sqrt{y}} \frac{1}{\sqrt{y-1}} & \frac{1}{2} \frac{1}{\sqrt{y-1}} & \frac{1}{2 \sqrt{y}} & 0 \\
1 & 0 & 0 & -\frac{1+y}{2 \sqrt{y}} & \xi
\end{array}\right),
$$

where $y \equiv a_{1}^{2} / a_{2}^{2}>1$ and $\xi$ is again an arbitrary nonvanishing real parameter, one finds that $K_{0}=S_{3}^{T} K S_{3}$ and $M_{0}=S_{3}^{T} M S_{3}$ are given by

$$
\begin{aligned}
K_{0} & =\left(\begin{array}{ccccc}
1 & 0 & 0 & 0 & 0 \\
0 & -1 & 0 & 0 & 0 \\
0 & 0 & 1 & 0 & 0 \\
0 & 0 & 0 & -1 & 0 \\
0 & 0 & 0 & 0 & 0
\end{array}\right) \text { and } \\
M_{0} & =\left(\begin{array}{ccccc}
0 & 0 & 0 & 0 & 0 \\
0 & -1 / a_{1}^{2} & 0 & 0 & 0 \\
0 & 0 & 1 / a_{2}^{2} & 0 & 0 \\
0 & 0 & 0 & 0 & -\frac{\xi}{a_{1} a_{2}} \\
0 & 0 & 0 & -\frac{\xi}{a_{1} a_{2}} & 0
\end{array}\right) .
\end{aligned}
$$

The two-by-two block in the lower-right of $K_{0}$ and $M_{0}$ cannot be simultaneously diagonalized, and also depends on an undetermined parameter $\xi$. Both are indications that this block corresponds to degrees of freedom that are unphysical. The field $\Psi_{5}$ only appears linearly through $M_{0}$ and it can be functionally integrated, leaving a functional delta function that sets $\Psi_{4}$ equal to zero. As before, integrating out $N-1$ fields (in this case $\Psi_{4}$ and $\Psi_{5}$ ) we are left with fields that represent the physical degrees of freedom expected in the Lee-Wick form of the theory, i.e., two Lee-Wick partners, one of which is a ghost. The mass spectrum again matches our expectation from the higherderivative form of the theory (see also Ref. [7]). In analogy to our previous results, the form of $S_{3}$ ensures that the potential $V\left(\phi_{1}\right)$ has no dependence on the auxiliary fields $\Psi_{4}$ and $\Psi_{5}$.

\section{General structure}

Based on our gained intuition, let us now make some more general comments applicable to general $N$. In our loop calculation discussed in the next section, we will focus on the mass renormalization of the field $\Psi_{1}$. As it turns out, it is most easily determined by working in the higherderivative form of the theory for $\phi_{1}$, and by extracting the part of this field on external lines that corresponds to the lightest physical mass eigenstate $\Psi_{1}$. Based on Eqs. (3.3) and (3.6) we see that this part is easily identified as having unit coefficient, i.e., $\phi_{1}=\Psi_{1}+\cdots$. This fact is true for arbitrary $N$, which can be verified by considering the partial fraction decomposition of the higher-derivative propagator for nondegenerate $a_{j}$,

$$
\frac{i}{p^{2}} \prod_{j=1}^{N-1}\left(1-a_{j}^{2} p^{2}\right)^{-1}=\frac{i}{p^{2}} b_{0}+\sum_{k=1}^{N-1} \frac{i}{p^{2}-1 / a_{k}^{2}} b_{k},
$$

which holds algebraically for 
$b_{0}=1 \quad$ and $\quad b_{k}= \begin{cases}-1 & \text { for } N=2 \\ -\prod_{\substack{j=1 \\ j \neq k}}^{N-1}\left(1-a_{j}^{2} / a_{k}^{2}\right)^{-1} & \text { for } N \geq 3 .\end{cases}$

The wave function renormalization of the lightest mass eigenstate, i.e., $b_{0}=1$, ensures that a self-energy diagram with two external $\phi_{1}$ lines and one with two external $\Psi_{1}$ lines are related by a relative factor of +1 . We also note in passing that the $b_{k}$ above are in agreement with the square of the elements of the first rows of $S_{2}$ and $S_{3}$, respectively, providing a useful consistency check.

With the basic structures in the finite- $N$ theory understood, let us now apply these techniques to study the quadratic divergences in such a theory.

\section{QUADRATIC DIVERGENCES}

As we remarked in the previous section, the field $\Psi_{1}$ represents the lightest physical state, and it is interesting to consider its mass renormalization, generated by a suitable self-interaction $V\left(\phi_{1}\right)$. We shall work in the higher-derivative theory (2.7) where we choose a quartic self-interaction

$$
V\left(\phi_{1}\right)=\frac{\lambda}{4 !} \phi_{1}^{4} .
$$

At one-loop, the self-energy for the $\phi_{1}$ field, $M^{2}\left(k^{2}\right)$, is given by

$$
\begin{aligned}
-i M^{2}\left(k^{2}\right) & =-i \frac{\lambda}{2} \int \frac{\mathrm{d}^{4} p}{(2 \pi)^{4}} D_{F}\left(p^{2}\right) \\
& =\frac{\lambda}{2} \int \frac{\mathrm{d}^{4} p}{(2 \pi)^{4}} \frac{1}{p^{2}} \prod_{j=1}^{N-1}\left(1-a_{j}^{2} p^{2}\right)^{-1},
\end{aligned}
$$

where $k$ is the external line momentum and we have inserted the higher-derivative propagator (2.8). The two external $\phi_{1}$ lines can be reexpressed in terms of the treelevel mass eigenstates $\Psi_{j}$, using the type of decomposition described in the previous section. We focus here on the case of two external $\Psi_{1}$ lines, which does not alter any numerical factors in Eq. (4.2); mixing effects induced at one-loop between $\Psi_{1}$ and $\Psi_{j}$, for $j>1$, become negligible in the limit of interest, i.e., where the tree-level masses are taken such that latter states are decoupled. It is our goal to compute this self-energy for finite $N$, as well as the asymptotic form of this result as $N \rightarrow \infty$. In what follows, we will employ the notation $m_{j}^{2}=1 / a_{j}^{2}$ and assume that all masses are nondegenerate. As shown in the previous section, these masses correspond to the poles of the higher-derivative propagator, and match those of the Lee-Wick theory as well, in both cases appearing with residues of alternating sign.
For an $N$ that is finite but not large, we will see that the self-energy scale is set by the smallest of the Lee-Wick partner masses $m_{j}^{2}$, as expected. This means that protecting the lightest scalar mass from large radiative corrections would not allow us to decouple the spectrum of Lee-Wick partner states. A different conclusion is reached when $N$ becomes large, as we will show by constructing an exactly solvable, nondegenerate mass parametrization that allows us to perform the $N \rightarrow \infty$ limit analytically. In this case, we will prove that the one-loop contribution to the scalar mass squared is set by the nonlocal scale $M_{\mathrm{nl}}^{2} \equiv 1 / \ell^{2}$, where

$$
M_{\mathrm{nl}}^{2} \sim \mathcal{O}\left(\frac{m_{1}^{2}}{N}\right)
$$

where $m_{1}$ is the mass of the lightest Lee-Wick resonance. This provides a mechanism of achieving a hierarchical separation of scales.

\section{A. Partial fraction method at finite $N$}

We first note that the higher-derivative propagator $D_{F}\left(p^{2}\right)$ in Eq. (4.2) has the partial fraction decomposition given in Eq. (3.8). Each term of the right-hand side is of a form suitable for Wick rotation, without any unusual prescriptions required in Lee-Wick theories for more complicated denominators [3]. As a result, we can do the same on the left-hand side and study the self-energy of interest by working in Euclidean space from the start $\left(p^{0} \rightarrow i p_{E}^{0}\right.$ and $\left.p^{2} \rightarrow-p_{E}^{2}\right)$. Hence,

$$
\begin{aligned}
-i M^{2}\left(k^{2}\right) & =-\frac{i \lambda}{2} \int \frac{\mathrm{d}^{4} p_{E}}{(2 \pi)^{4}} \frac{1}{p_{E}^{2}} \prod_{j=1}^{N-1}\left(1+a_{j}^{2} p_{E}^{2}\right)^{-1} \\
& =-\frac{i \lambda}{32 \pi^{2}} \int_{0}^{\infty} \mathrm{d} u \prod_{j=1}^{N-1}\left(1+a_{j}^{2} u\right)^{-1}
\end{aligned}
$$

where we have defined $u \equiv p_{E}^{2}$. The expression on the right of Eq. (4.4) can be written in terms of partial fractions. We assume $N \geq 3$ and find

$\prod_{j=1}^{N-1}\left(1+a_{j}^{2} u\right)^{-1}=\sum_{j=1}^{N-1} \frac{d_{j}}{1+a_{j}^{2} u} \quad$ with $\quad d_{k}=\prod_{\substack{j=1 \\ j \neq k}}^{N-1} \frac{a_{k}^{2}}{a_{k}^{2}-a_{j}^{2}}$,

which allows easy integration of each term in the sum; this leaves us with

$$
-i M^{2}\left(k^{2}\right)=\frac{i \lambda}{32 \pi^{2}} \sum_{k=1}^{N-1} d_{k} m_{k}^{2} \ln m_{k}^{2}, \text { where } d_{k}=\prod_{\substack{j=1 \\ j \neq k}}^{N-1} \frac{m_{j}^{2}}{m_{j}^{2}-m_{k}^{2}} .
$$


Here we have expressed the result in terms of the resonance masses, and we have used at an intermediate step that

$$
\sum_{k=1}^{N-1} d_{k} m_{k}^{2}=0
$$

which holds for $N \geq 3$. For $N=2$, no such cancellation can occur and one is instead left with a result that diverges logarithmically with the cutoff. In the case where $N=3$ one finds

$$
M^{2}\left(k^{2}\right)=\frac{\lambda}{32 \pi^{2}} \frac{m_{1}^{2} m_{2}^{2}}{m_{2}^{2}-m_{1}^{2}} \ln \left(\frac{m_{2}^{2}}{m_{1}^{2}}\right) .
$$

Notice that if $m_{2} \gg m_{1}$, the radiative correction to the bare mass squared is positive and with magnitude set by the lightest Lee-Wick partner mass $m_{1}^{2}$. The same is true for larger $N$, provided we are far from the asymptotic case considered below; when the additional states beyond the first three are made heavy they decouple, leaving the same $N=3$ effective theory, so that the scale of the result is again set by $m_{1}^{2}$ when $m_{2}^{2}$ is also made heavy.

Last, let us note that the general expression in Eq. (4.6) is valid for an arbitrary choice of nondegenerate $m_{j}$. This will be of use in confirming the results of the next subsection, where we consider the limiting form for the self-energy as $N \rightarrow \infty$ that follows from a specific parametrization of the spectrum.

\section{B. Exactly solvable model at infinite $N$}

We expect asymptotic nonlocality to be obtained in the theory defined by Eq. (2.7) if the Lee-Wick masses approach a common value as they are simultaneously decoupled and their number is taken to infinity. Here we implement a concrete ansatz that allows us to achieve this result and to arrive at a closed-form expression for the scalar self-energy. Consider the parametrization

$$
a_{j}^{2}=\left(2-\frac{j}{N}\right) \frac{2 \ell^{2}}{3 N}
$$

where $\ell$ denotes the scale of nonlocality. For algebraic convenience we use factors of $N$ rather than $N-1$ in this parametrization of $a_{j}$, which reproduces identical large- $N$ asymptotics. For the masses, this parametrization amounts to

$$
m_{j}^{2}=\frac{3}{2} \frac{1}{2-\frac{j}{N}} N M_{\mathrm{nl}}^{2}
$$

consistent with Eq. (4.3). This parametrization provides for a nondegenerate mass spectrum, for any finite $N$, a desired feature we discussed earlier. In Fig. 1 we plot the first few mass values $m_{j}^{2}$ for different values of $N$.

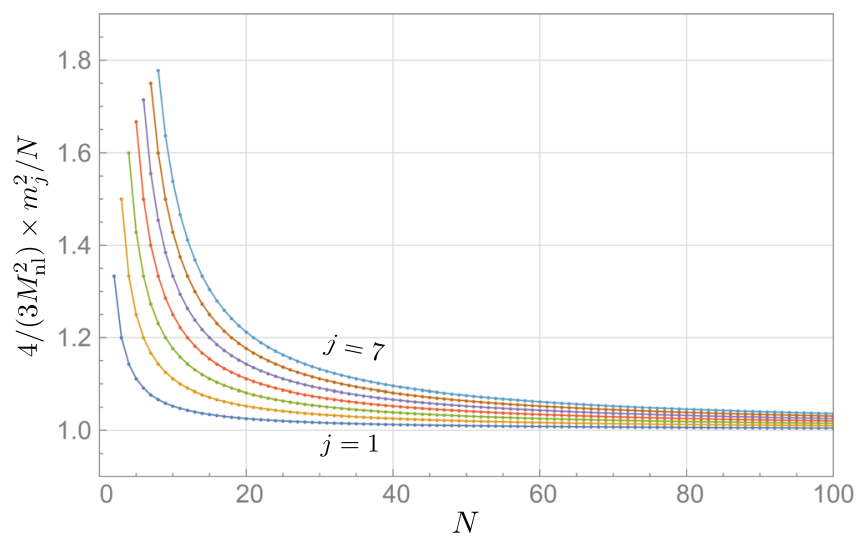

FIG. 1. We plot the first seven masses for the parametrization (4.10), rescaled by a factor of $N$, for $j=1$ to 7 (bottom to top). In the large- $N$ limit, all masses tend to a common large Lee-Wick value (here normalized to unity).

Equation (4.9) allows us to evaluate the product in Eq. (4.4) directly. One can prove the identity

$\prod_{j=1}^{N-1}\left(1+a_{j}^{2} p_{E}^{2}\right)=\left(\frac{2 \ell^{2} p_{E}^{2}}{3 N^{2}}\right)^{N-1} P\left(1+N+\frac{3 N^{2}}{2 \ell^{2} p_{E}^{2}}, N-1\right)$,

where $P(a, n)$ is the Pochhammer symbol (sometimes also called "rising factorial") given by

$$
P(a, n) \equiv(a)_{n} \equiv \frac{\Gamma(a+n)}{\Gamma(a)} .
$$

In the limiting case of large $N$ we may employ the Stirling identity,

$$
\Gamma(N+1) \approx \sqrt{2 \pi N}\left(\frac{N}{e}\right)^{N}
$$

and after some manipulations arrive at

$$
\begin{aligned}
& \lim _{N \rightarrow \infty} \prod_{j=1}^{N-1}\left(1+a_{j}^{2} p_{E}^{2}\right) \\
& =e^{\ell^{2} p_{E}^{2}}\left[1-\left(1+\frac{14}{27} p_{E}^{2} \ell^{2}\right) \frac{p_{E}^{2} \ell^{2}}{N}+\mathcal{O}\left(\frac{1}{N^{2}}\right)\right] .
\end{aligned}
$$

The limit point corresponds to a nonlocal exponential propagator, as one could have expected from previous considerations. However, our calculations prove that for nondegenerate masses one recovers the exponential propagator as well, which one may regard as a nontrivial consistency check. Perhaps more importantly, this calculation demonstrates the emergence of the nonlocal scale as one appropriately decouples an infinite number of 
Lee-Wick resonances, i.e., how asymptotic nonlocality is achieved.

As a side note, it is well known that analytic continuation in the presence of nonlocal operators of the form $e^{\ell^{2} \square}$ is nontrivial due to the existence of essential singularities in the complex momentum plane for such entire functions [46]. Our construction sidesteps these difficulties. For finite $N$ we simply have a Lee-Wick theory in which suitable prescriptions like those of Cutkosky, Landshoff, Olive, and Polkinghorne can presumably be used to guarantee the proper behavior of the quantum theory [3]; see also [4].

At any rate, the subsequent integration over the Euclidean momentum can be performed analytically and one finds the following simple result for the self-energy:

$\lim _{N \rightarrow \infty} M^{2}\left(k^{2}\right)=\frac{\lambda}{16 \pi^{2}} \int_{0}^{\infty} \mathrm{d} p_{E} p_{E} e^{-\ell^{2} p_{E}^{2}}=\frac{\lambda}{32 \pi^{2} \ell^{2}}=\frac{\lambda M_{\mathrm{nl}}^{2}}{32 \pi^{2}}$.

Note that this result is finite and its scale is set purely by $\ell$, as opposed to the finite-and-not-so-large $N$ case where the scale is set by the lightest Lee-Wick resonance mass. In other words, the large- $N$ limit has provided us with a hierarchical separation between the scale of the lightest Lee-Wick resonance and the potentially much lighter nonlocal scale. This suggests an interesting approach to evading experimental constraints on new particles in more realistic higher-derivative theories that are designed to address the hierarchy problem.

\section{Large- $N$ comparison}

One may wonder whether the different approaches we have presented in Secs. IVA and IV B agree with one another. In other words, do the $d_{k}$ prefactors in Eq. (4.6), computed in the higher-derivative theory at finite $N$, conspire to give the nonlocal scale that emerges in Eq. (4.15) in the large- $N$ limit? The analytic calculations would imply that this the case, and upon substituting our mass parametrization (4.10) into the exact result (4.6) we can confirm this correspondence numerically as well. This is shown in Fig. 2.

Having the general result (4.6) at our disposal, we may also probe different mass parametrizations. For example, the parametrization

$$
a_{j}^{2}=\left(1+\frac{(N-j)^{b}}{N^{b}}\right) \frac{b+1}{b+2} \frac{\ell^{2}}{N}, \quad b \in \mathbb{N}
$$

also reproduces the limit (4.15). This supports the notion that (i) our results are independent of how exactly one chooses to lift the degeneracy in the Lee-Wick spectrum, and (ii) that the relevant scale is indeed the asymptotically nonlocal scale. The only two requirements on the mass functions $m_{j}$ seem to be nondegeneracy as well as the

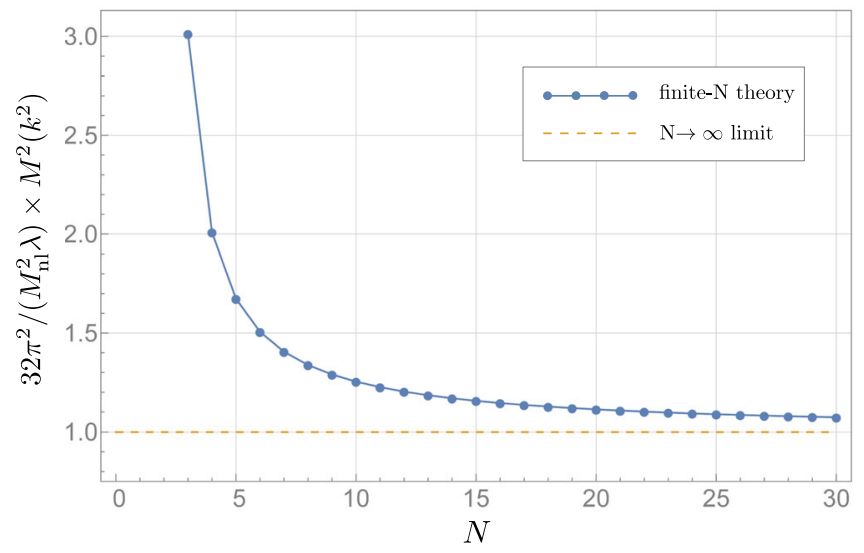

FIG. 2. For our parametrization (4.10) we compare the selfenergy at finite $N$ (solid line), given by Eq. (4.6), to the value obtained in the $N \rightarrow \infty$ limit (dashed line), given by Eqs. (4.14) and (4.15). It is clear that for large $N$ the results converge.

scaling behavior that $m_{j}^{2} \sim N$ as $N \rightarrow \infty$. It is interesting to ponder the ultraviolet completions that would lead to higher-derivative theories with similar spectra; however, this would likely require an understanding of Planckscale physics that goes beyond a quantum field theory description.

The numerical results for the one-loop amplitude that we have computed in our local, higher-derivative theory demonstrate that the regulator of loop diagrams in the limit of large but finite $N$ is well approximated by the nonlocal scale that appears in the limiting form of the theory. We expect that the scale that regulates divergences in higher-loop diagrams is no different, based on a dimensional argument: in the large- $N$ limit, the Lagrangian approaches a form where the nonlocal scale is the only one available to regulate divergences, and no small dimensionless factors appear in the theory. This implies that our results should persist in higher-order perturbative calculations, though we do not consider those in the present work.

\section{CONCLUSIONS}

Higher-derivative quadratic terms have received substantial attention as a means of obtaining improved ultraviolet behavior of loop amplitudes. When these terms are of finite order in the number of derivatives, as in the Lee-Wick standard model [2], additional poles in the two-point function appear that correspond to new particles; these might be expected at the $\mathrm{TeV}$ scale if they participate in a solution to the hierarchy problem. So far, no such states have been observed experimentally. On the other hand, in theories with higher-derivative quadratic terms of infinite order, as in models where the $\square$ operator appears as the argument of an entire function, there are no new particles, but there are other complications: the simplest formulation of such nonlocal theories in Minkowski spacetime violate 


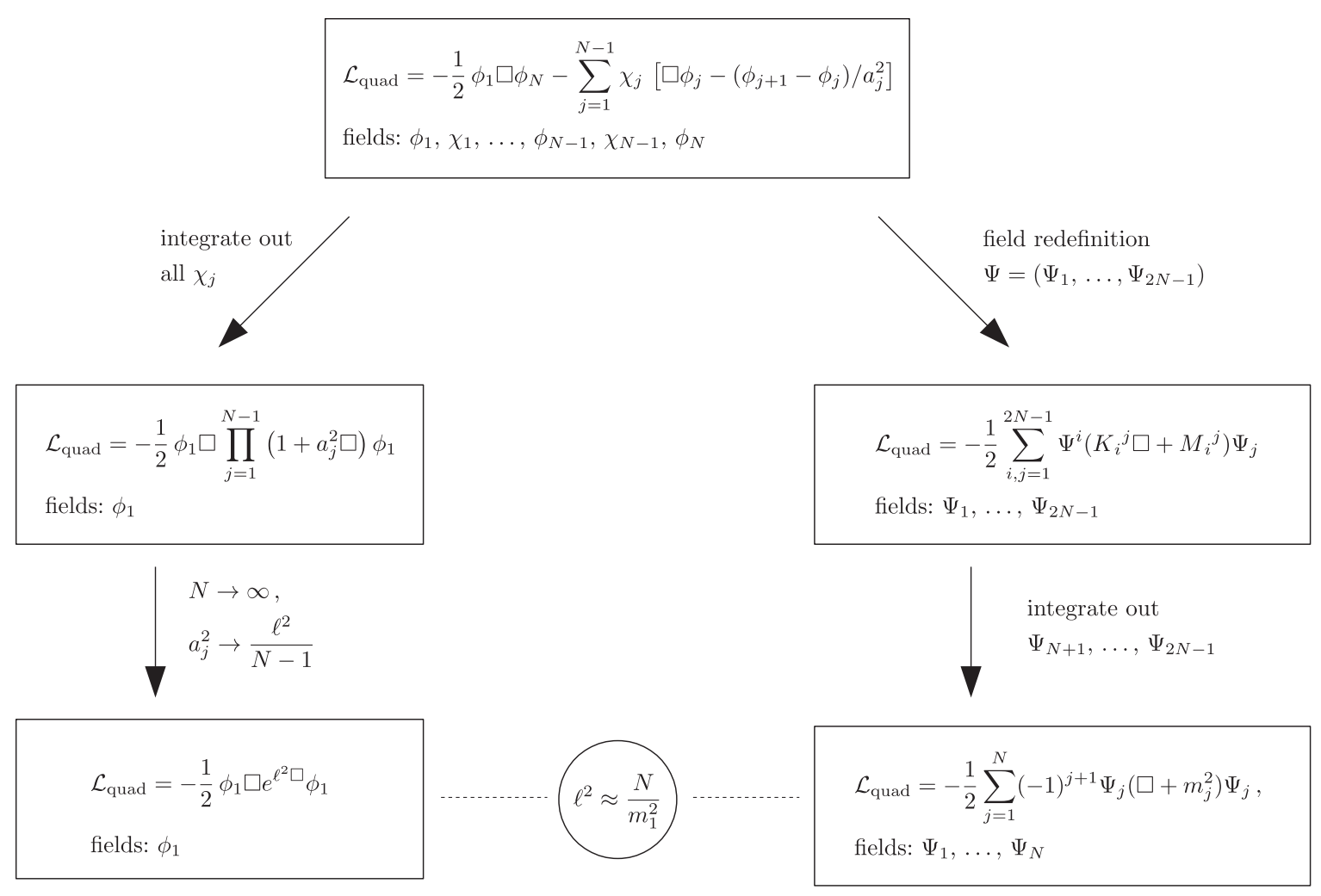

FIG. 3. A schematic overview of the quadratic terms of the different theories developed in this paper, and how they are interrelated. In the large- $N$ limit, the Lee-Wick resonances tend to infinity, whereas the scale of nonlocality $\ell$ remains finite.

unitarity [29,31-34], a problem related to the fact that there are directions in the complex $p^{0}$ plane where loop integrands diverge. Another technical complication lies in the fact that entire functions typically possess essential singularities in the complex plane, making a Wick rotation considerably more involved. While several ways have been proposed to avoid this problem in nonlocal theories [46], we present a different approach to the problem in the present work: formulating a higher-derivative theory with $N$ propagator poles that can be made unitary following the same prescriptions as in conventional Lee-Wick theories, it approaches the desired nonlocal form (the exponential of a differential operator) as $N \rightarrow \infty$. We call this "asymptotic nonlocality." At the same time, the mass spectrum of Lee-Wick partners is decoupled in a specific way. Even away from the $N \rightarrow \infty$ limit, the effective regulator in loop amplitudes is set by the would-be nonlocal scale, which can be hierarchically separated from the scale of the lightest Lee-Wick partner. In the present work, we demonstrate this via a simple scalar model and develop a theory of auxiliary fields that relates the higher-derivative and Lee-Wick forms of the theory; see Fig. 3 for a schematic overview. It is known that the Lee-Wick approach may be generalized to gauge theories, as has been shown in the Lee-Wick standard model [2], as well as in a higher-derivative extension thereof involving more than one Lee-Wick partner particle [7]. We will study the generalization of the present work to spontaneously broken gauge theories and fields of other spin in a separate publication.

There are other issues that are also worthy of further investigation. The form of our theory is nongeneric, as would be expected since the form of the (well-studied) nonlocal theory that it is designed to match when $N \rightarrow \infty$ is also nongeneric. It would be worthwhile to study the renormalization of the theory, how it corresponds to the renormalization of the nonlocal theory, and whether this leads to any qualitative changes in the properties of amplitudes. It would also be interesting to construct the argument for power-counting renormalizability for gauge theories in our auxiliary-field construction for arbitrary $N$, similar to what has been achieved for conventional LeeWick theories. A generalization to asymptotically nonlocal gravity would also be of interest. The asymptotically nonlocal model presented here provides a convenient interpolation between conventional Lee-Wick theories and nonlocal quantum field theories, while keeping the best features of both. We look forward to investigating theories of this type in a variety of contexts in future work.

\section{ACKNOWLEDGMENTS}

We thank the NSF for support under Grant No. PHY1819575. 
[1] T. D. Lee and G. C. Wick, Negative metric and the unitarity of the S-matrix, Nucl. Phys. B9, 209 (1969); Finite theory of quantum electrodynamics, Phys. Rev. D 2, 1033 (1970).

[2] B. Grinstein, D. O'Connell, and M. B. Wise, The Lee-Wick standard model, Phys. Rev. D 77, 025012 (2008).

[3] R. E. Cutkosky, P. V. Landshoff, D. I. Olive, and J. C. Polkinghorne, A non-analytic S-matrix, Nucl. Phys. B12, 281 (1969).

[4] D. Anselmi and M. Piva, A new formulation of Lee-Wick quantum field theory, J. High Energy Phys. 06 (2017) 066.

[5] B. Grinstein, D. O'Connell, and M. B. Wise, Causality as an emergent macroscopic phenomenon: The Lee-Wick $\mathrm{O}(\mathrm{N})$ model, Phys. Rev. D 79, 105019 (2009).

[6] C. D. Carone and R. F. Lebed, Minimal Lee-Wick extension of the standard model, Phys. Lett. B 668, 221 (2008).

[7] C. D. Carone and R. F. Lebed, A higher-derivative LeeWick standard model, J. High Energy Phys. 01 (2009) 043.

[8] T. G. Rizzo, Searching for Lee-Wick gauge bosons at the LHC, J. High Energy Phys. 06 (2007) 070; J. R. Espinosa, B. Grinstein, D. O'Connell, and M. B. Wise, Neutrino masses in the Lee-Wick standard model, Phys. Rev. D 77, 085002 (2008); T. R. Dulaney and M. B. Wise, Flavor changing neutral currents in the Lee-Wick standard model, Phys. Lett. B 658, 230 (2008); F. Krauss, T. E. J. Underwood, and R. Zwicky, The process $g g \rightarrow h_{0} \rightarrow \gamma \gamma$ in the Lee-Wick standard model, Phys. Rev. D 77, 015012 (2008); Erratum, Phys. Rev. D 83, 019902 (2011); Z. Fodor, K. Holland, J. Kuti, D. Nogradi, and C. Schroeder, New Higgs physics from the lattice, Proc. Sci., LATTICE2007 (2007) 056 [arXiv:0710.3151]; T. G. Rizzo, Unique identification of Lee-Wick gauge bosons at linear colliders, J. High Energy Phys. 01 (2008) 042; E. Alvarez, L. Da Rold, C. Schat, and A. Szynkman, Electroweak precision constraints on the Lee-Wick standard model, J. High Energy Phys. 04 (2008) 026; T. E. J. Underwood and R. Zwicky, Electroweak precision data and the Lee-Wick standard model, Phys. Rev. D 79, 035016 (2009); B. Fornal, B. Grinstein, and M. B. Wise, Lee-Wick theories at high temperature, Phys. Lett. B 674, 330 (2009); C. D. Carone and R. Primulando, Constraints on the Lee-Wick Higgs sector, Phys. Rev. D 80, 055020 (2009); C. D. Carone, R. Ramos, and M. Sher, LHC constraints on the Lee-Wick Higgs sector, Phys. Lett. B 732, 122 (2014); A. Accioly, P. Gaete, J. Helayel-Neto, E. Scatena, and R. Turcati, Investigations in the Lee-Wick electrodynamics, Mod. Phys. Lett. A 26, 1985 (2011); T. Figy and R. Zwicky, The other Higgses, at resonance, in the Lee-Wick extension of the standard model, J. High Energy Phys. 10 (2011) 145.

[9] P. A. Zyla et al. (Particle Data Group), Review of particle physics, Prog. Theor. Exp. Phys. 2020, 083 C01 (2020).

[10] G. V. Efimov, Nonlocal quantum theory of the scalar field, Commun. Math. Phys. 5, 42 (1967).

[11] N. V. Krasnikov, Nonlocal gauge theories, Theor. Math. Phys. 73, 1184 (1987).

[12] Yu. V. Kuz'min, Convergent nonlocal gravitation, Sov. J. Nucl. Phys. 50, 1011 (1989).

[13] E. T. Tomboulis, Super-renormalizable gauge and gravitational theories, arXiv:hep-th/9702146.

[14] L. Modesto, Super-renormalizable quantum gravity, Phys. Rev. D 86, 044005 (2012).
[15] T. Biswas, E. Gerwick, T. Koivisto, and A. Mazumdar, Towards Singularity and Ghost Free Theories of Gravity, Phys. Rev. Lett. 108, 031101 (2012).

[16] G. Wataghin, Bemerkung ber die Selbstenergie der Elektronen (Engl. transl. A note on the self-energy of electrons), Z. Phys. 88, 92 (1934).

[17] W. Pauli and F. Villars, On the invariant regularization in relativistic quantum theory, Rev. Mod. Phys. 21, 434 (1949).

[18] A. Pais and G. Uhlenbeck, On field theories with nonlocalized action, Phys. Rev. 79, 145 (1950).

[19] E. Witten, Noncommutative geometry and string field theory, Nucl. Phys. B268, 253 (1986).

[20] P. H. Frampton and Y. Okada, Effective scalar field theory of p-adic string, Phys. Rev. D 37, 3077 (1988).

[21] A. A. Tseytlin, On singularities of spherically symmetric backgrounds in string theory, Phys. Lett. B 363, 223 (1995).

[22] W. Siegel, Stringy gravity at short distances, arXiv:hep-th/ 0309093.

[23] T. Biswas, A. Mazumdar, and W. Siegel, Bouncing universes in string-inspired gravity, J. Cosmol. Astropart. Phys. 03 (2006) 009.

[24] G. Calcagni and L. Modesto, Nonlocality in string theory, J. Phys. A 47, 355402 (2014).

[25] G. Calcagni and L. Modesto, Nonlocal quantum gravity and M-theory, Phys. Rev. D 91, 124059 (2015).

[26] J. Edholm, A. S. Koshelev, and A. Mazumdar, Behavior of the Newtonian potential for ghost-free gravity and singularity-free gravity, Phys. Rev. D 94, 104033 (2016).

[27] J. Boos, V. P. Frolov, and A. Zelnikov, Gravitational field of static $p$-branes in linearized ghost-free gravity, Phys. Rev. D 97, 084021 (2018).

[28] B. L. Giacchini and T. de Paula Netto, Effective delta sources and regularity in higher-derivative and ghost-free gravity, J. Cosmol. Astropart. Phys. 07 (2019) 013.

[29] E. T. Tomboulis, Renormalization and unitarity in higherderivative and nonlocal gravity theories, Mod. Phys. Lett. A 30, 1540005 (2015).

[30] I. L. Shapiro, Counting ghosts in the 'ghost-free' nonlocal gravity, Phys. Lett. B 744, 67 (2015).

[31] C. D. Carone, Unitarity and microscopic acausality in a nonlocal theory, Phys. Rev. D 95, 045009 (2017).

[32] F. Briscese and L. Modesto, Cutkosky rules and perturbative unitarity in Euclidean nonlocal quantum field theories, Phys. Rev. D 99, 104043 (2019).

[33] F. Briscese and L. Modesto, Nonunitarity of Minkowskian nonlocal quantum field theories, arXiv:2103.00353.

[34] A. S. Koshelev and A. Tokareva, Unitarity of Minkowski nonlocal theories made explicit, arXiv:2103.01945 [Phys. Rev. D (to be published)].

[35] L. Buoninfante, G. Lambiase, and A. Mazumdar, Ghost-free infinite-derivative quantum field theory, Nucl. Phys. B944, 114646 (2019).

[36] J. Boos, Effects of nonlocality in gravity and quantum theory, Ph.D. thesis, University of Alberta, 2020.

[37] L. Buoninfante, A. Ghoshal, G. Lambiase, and A. Mazumdar, Transmutation of nonlocal scale in infinite-derivative field theories, Phys. Rev. D 99, 044032 (2019).

[38] N. Arkani-Hamed, A. G. Cohen, and H. Georgi, (De) constructing Dimensions, Phys. Rev. Lett. 86, 4757 (2001). 
[39] C. T. Hill, S. Pokorski, and J. Wang, Gauge invariant effective Lagrangian for Kaluza-Klein modes, Phys. Rev. D 64, 105005 (2001).

[40] G. Calcagni, M. Montobbio, and G. Nardelli, Localization of nonlocal theories, Phys. Lett. B 662, 285 (2008).

[41] G. Calcagni and G. Nardelli, Nonlocal gravity and the diffusion equation, Phys. Rev. D 82, 123518 (2010).

[42] N. Kan, M. Kuniyasu, K. Shiraishi, and Z. Wu, Discrete heat kernel, UV modified Green's function, and higherderivative theories, arXiv:2007.00220.
[43] V. P. Frolov and A. Zelnikov, Radiation from an emitter in the ghost-free scalar theory, Phys. Rev. D 93, 105048 (2016).

[44] J. Boos, V. P. Frolov, and A. Zelnikov, Probing the vacuum fluctuations in scalar ghost-free theories, Phys. Rev. D 99, 076014 (2019).

[45] C. D. Carone, Aspects and applications of nonlocal Lorentzviolation, Phys. Rev. D 102, 095006 (2020).

[46] R. Pius and A. Sen, Cutkosky rules for superstring field theory, J. High Energy Phys. 10 (2016) 024; Erratum, J. High Energy Phys. 09 (2018) 122. 